\title{
BMJ Open Intracytoplasmic sperm injection (ICSI) versus conventional in vitro fertilisation (IVF) in couples with non-severe male infertility (NSMI-ICSI): protocol for a multicentre randomised controlled trial
}

Danni Zheng, ${ }^{1,2,3,4,5,6}$ Lin Zeng (10 , ${ }^{7}$ Rui Yang, ${ }^{1,2,3,4,5,6}$ Ying Lian, ${ }^{1,2,3,4,5,6}$ Yi-Min Zhu, ${ }^{8}$ Xiaoyan Liang, ${ }^{9}$ Li Tang, ${ }^{10}$ Huichun Wang, ${ }^{11}$ Yunxia Cao, ${ }^{12}$ Guimin Hao, ${ }^{13}$ Jianqiao Liu, ${ }^{14}$ Junli Zhao, ${ }^{15}$ Rui Wang, ${ }^{16,17}$ Ben Willem Mol, ${ }^{17}$ Rong Li, ${ }^{1,2,3,4,5,6}$ He-Feng Huang, ${ }^{18}$ Jie Qiao ${ }^{1,2,3,4,5,6}$

To cite: Zheng D, Zeng $L$, Yang R, et al. Intracytoplasmic sperm injection (ICSI) versus conventional in vitro fertilisation (IVF) in couples with non-severe male infertility (NSMI-ICSI): protocol for a multicentre randomised controlled trial. BMJ Open 2019;9:e030366. doi:10.1136/ bmjopen-2019-030366

- Prepublication history for this paper is available online. To view these files, please visit the journal online (http://dx.doi. org/10.1136/bmjopen-2019030366).

$\mathrm{DZ}, \mathrm{LZ}$ and $\mathrm{RY}$ are joint first authors.

Received 11 March 2019 Revised 19 June 2019 Accepted 23 August 2019

Check for updates

(c) Author(s) (or their employer(s)) 2019. Re-use permitted under CC BY-NC. No commercial re-use. See rights and permissions. Published by BMJ.

For numbered affiliations see end of article.

Correspondence to Dr He-Feng Huang; huanghefg@hotmail.com

Dr Jie Qiao; jie.qiao@263.net

\section{ABSTRACTS}

Introduction Intracytoplasmic sperm injection (ICSI), originally introduced as add-on to in vitro fertilisation (IVF) for couples with severe male infertility, is in current clinical practice also used in couples with mild male or even unexplained infertility. However, ICSI has involved unresolved concerns regarding the selection and damage to gametes and the health conditions of the offspring, and it is also labour intensive and therefore more expensive than conventional IVF. High-quality well-powered randomised clinical trials (RCTs) comparing ICSI and IVF are lacking.

Methods and analysis We propose a multicentre, openlabel RCT in 10 reproductive medical centres across China. We will study couples with non-severe male infertility (defined as a semen concentrate $5-15 \times 10^{6} / \mathrm{mL}$ or sperm with a progressive motility $10 \%-32 \%$ ) scheduled for their first or second ICSI or IVF cycle, as low fertility rate after fertilisation are more frequent in this population, which could lead to controversy about ICSI or conventional IVF for fertilisation. On the day of oocyte retrieval, eligible participants are after informed consent be randomised to undergo either ICSI or conventional IVF in a 1:1 treatment ratio. Other standard assisted reproductive treatments are similar and parallel between two groups. Our primary outcome is ongoing pregnancy leading to live birth after the first cycle with embryo transfer. To demonstrate or refute a difference of $7 \%$ between ICSI and conventional IVF, we need to include 2346 women (1173 in each intervention arm). In addition, we will follow-up neonatal outcomes after delivery to identify the influence of ICSI on offspring.

Ethics and dissemination Ethical approval was obtained from Peking University Third Hospital medical science research ethics committee. The findings will be disseminated to the public through conference presentations and peer-reviewed scientific journals. Trial registration number ClinicalTrials.gov registry (NCT03298633).

\section{INTRODUCTION}

Male infertility is caused by impaired sperm production and function due to different
Strengths and limitations of this study

- It is the first randomised controlled clinical trial with a large sample size comparing intracytoplasmic sperm injection (ICSI) and conventional invitro fertilisation (IVF) among patients with non-severe male infertility in 10 centres across China.

- This study will provide evidence on whether ICSI or conventional IVF is the better method for fertilisation in terms of live birth for non-severe male infertility.

- Range of sperm parameters (semen concentrate $5-15 \times 10^{6} / \mathrm{mL}$ or sperm with progressive motility $10-32 \%$ ) in our study based on the fifth edition of WHO manual will be applicable to couples with non-severe male infertility as many as possible.

- The sample size and power calculation were focused on the primary outcome of this study, with the limited power to detect other secondary outcomes.

congenital or acquired factors, ${ }^{1}$ and has been estimated to be associated with $\sim 30 \%$ of infertility. ${ }^{23}$ Assisted reproductive technology (ART) is perceived as a more successful treatment. ${ }^{45}$ Originally applied in women with tubal damage in 1970s, in vitro fertilisation (IVF) is now acknowledged as an effective treatment for infertility as a major component of ART. ${ }^{6}$ However, conventional IVF was much less effective when the semen characteristics were grossly below the standard values according to the WHO fourth edition sperm parameter values and when fertilisation rate in previous cycles was low. ${ }^{78}$

In $1992,{ }^{9}$ intracytoplasmic sperm injection (ICSI), a technique where a single spermatozoon was injected mechanically into an oocyte in vitro to achieve fertilisation, was introduced. While complete fertilisation 
failure was reported up to $50 \%$ of the conventional IVF treatments for couples with moderate male infertility (moderate oligozoospermia, asthenozoospermia and teratozoospermia), this occurred in $<3 \%$ of the couples undergoing ICSI. $^{10-13}$ Consequently, ICSI has been applied worldwide to treat severe male infertility. ${ }^{14-16}$

The high success rate of ICSI has resulted in its increased use in other populations for whom conventional IVF may be an option, particularly non-male factor infertility. In Europe, in 2012 ICSI was used in $69 \%$ of IVF cycles compared with $35 \%$ in 1997, while in the Middle East, South-America and South-East Asia, ICSI is performed in $100 \%$ of IVF cycles. ${ }^{17}{ }^{18}$ In the USA, between 1996 and 2012, the use of ICSI in IVF cycles has increased from $34 \%$ to $76 \%$. The greatest increase was documented in non-male factor infertility, where the use of ICSI went from $15 \%$ to $67 \%$ during this time period. ${ }^{19}$

There are concerns on the increased use of ICSI, as ICSI is time-consuming, expensive and involves unresolved concerns regarding the damage to gametes and the health conditions of the offspring. ${ }^{20-24}$ Many studies have indicated the routine use of ICSI in non-male factors infertility was not recommended to improve the clinical outcomes. ${ }^{25-28}$ For non-severe male factor infertility, including mild and moderate oligospermia with or without asthenospermia, the fertilisation and pregnancy outcome after ICSI compared with conventional IVF is unclear. Studies randomising sibling oocytes have shown conflicting results. Several studies have documented higher fertilisation rates and lower rates of fertilisation failure in these couples undergoing ICSI. ${ }^{29-31}$ Other studies did not support the benefit of ICSI in prevention of total fertilisation failure as there were no significant differences between ICSI and conventional IVF in embryo quality, implantation, clinical pregnancy or live birth rates. ${ }^{32-34}$ These studies have limitations such as small sample size, non-randomised couples or no evaluation of live births. In addition, fewer application of ICSI in China may result in low fertility rate for patients with non-severe male infertility, which would give raise to controversy about ICSI or conventional IVF for fertilisation during ART in these population. ${ }^{35}$

In view of this situation, we plan an adequately powered multicentre randomised controlled clinical trial to assess whether ICSI or conventional IVF is more effective in couples with non-severe male infertility.

\section{Methods and analysis}

\section{Study design}

We plan a multicentre, parallel, open-label, randomised controlled clinical trial (1:1 treatment ratio). The flowchart followed Standard Protocol Items: Recommendations for Interventional Trials checklist showing enrolment, allocation, treatment and follow-up of participants is presented in figure $1 .^{36}$ In addition, the schedule of enrolment, interventions and assessments during the study period is shown in table 1 .

\section{Study setting}

The study will recruit participants from 10 reproductive medical centres across China: Peking University Third Hospital, International Peace Maternity and Child Health Hospital of Shanghaijiao Tong University, Women's Hospital of Zhejiang University, The Sixth Affiliated Hospital of Sun Yat-Sen University, First Affiliated Hospital of Kunming Medical University, Haidian Maternal and Child Health Hospital, First Affiliated Hospital of Anhui Medical University, The Second Hospital of Hebei Medical University, The Third Affiliated Hospital of Guangzhou Medical University and General Hospital of Ningxia Medical University.

An independent data and safety monitoring board (DSMB), with members with clinical and statistical expertise, will monitor the trial progress and interim results at regular intervals.

\section{Eligibility criteria}

Couples presenting to reproductive medical centre of the involved hospitals will be screened for following eligibility to be enrolled in our trial.

\section{Inclusion criteria}

1. Infertile couples scheduled for their first or second IVF/ICSI cycle.

2. Male partner has non-severe male infertility, defined as a semen concentrate $5-15 \times 10^{6} / \mathrm{mL}$ or sperm with progressive motility (type a+b) $10 \%-32 \%$.

3. Women received either gonadotrophin-releasing hormone agonist (GnRH-a) protocol or gonadotrophin-releasing hormone antagonist (GnRH-ant) protocol as their controlled ovarian hyperstimulation $(\mathrm{COH})$ treatment.

4. Informed consent obtained.

\section{Exclusion criteria}

1. Couple with a contraindication for IVF or ICSI, including poorly controlled type 1 or type 2 diabetes mellitus; undiagnosed liver disease or dysfunction (based on serum liver enzyme test results); renal disease or abnormal serum renal function; anaemia; history of deep venous thrombosis, pulmonary embolus or cerebrovascular accident; uncontrolled hypertension or known symptomatic heart disease; history of (or suspected) cervical carcinoma, endometrial carcinoma or breast carcinoma; and unexplained colporrhagia.

2. Couples receiving donor sperm or donor eggs.

3. Couples undergoing preimplantation genetic testing.

4. Sperm concentration with progressive motility used for insemination $<0.1 \times 10^{6} / \mathrm{mL}$ on the day of oocyte retrieval.

5 . Women with 0 oocyte retrieved.

6. Using frozen semen.

7. Poor fertilisation in previous cycle $(\leq 25 \%)$.

In this study, couples with various female indications for IVF will be included. The sperm parameters defining non-severe male infertility are evaluated according to the WHO fifth edition sperm parameter values and the 

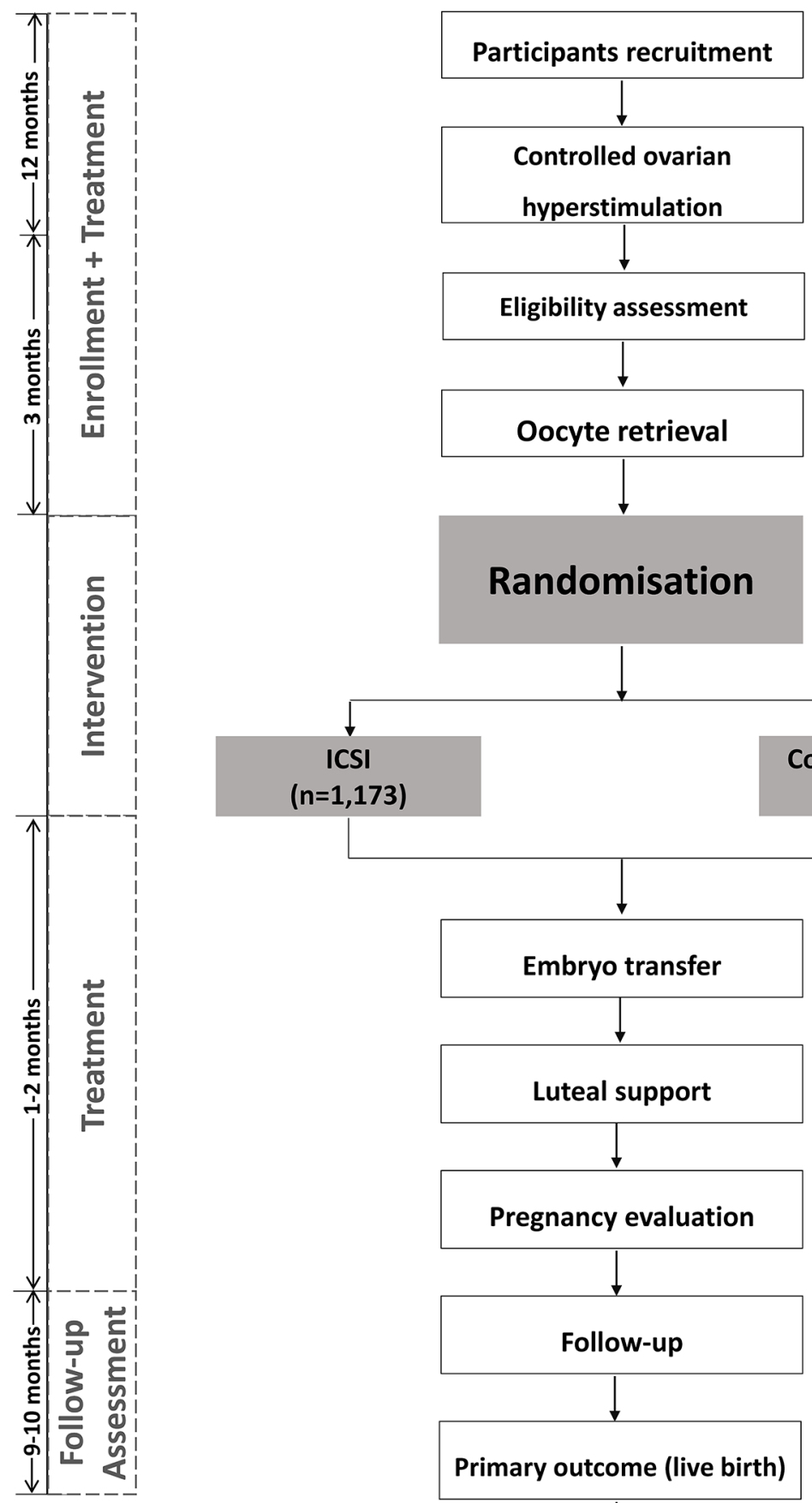

Figure 1 Flowchart followed SPIRIT checklist showing patient enrolment, allocation, treatment and follow-up of participants. ICSI, intracytoplasmic sperm injection; IVF, in vitro fertilisation; SPIRIT, Standard Protocol Items: Recommendations for Interventional Trials.

sperm parameters are subject to the latest sperm analysis. ${ }^{37}$ Participants have the right to decline participation during the whole process, and they can withdraw their consent at any time. Their consent or refusal to consent will not affect their conventional clinical treatments.

\section{Recruitment}

Infertile couples who come to the outpatient clinic or medical record of infertile couples who have received
$\mathrm{COH}$ treatment will be screened by a dedicated research team. Eligible couples will then, before oocyte retrieval, explained by a member of the research team the trial details. After this information, couples will be offered time for consideration to decide whether to participate in the trial. Couples who agree to participate will be asked to sign the consent form in their next scheduled visit. An individual record of all non-recruited patients and 


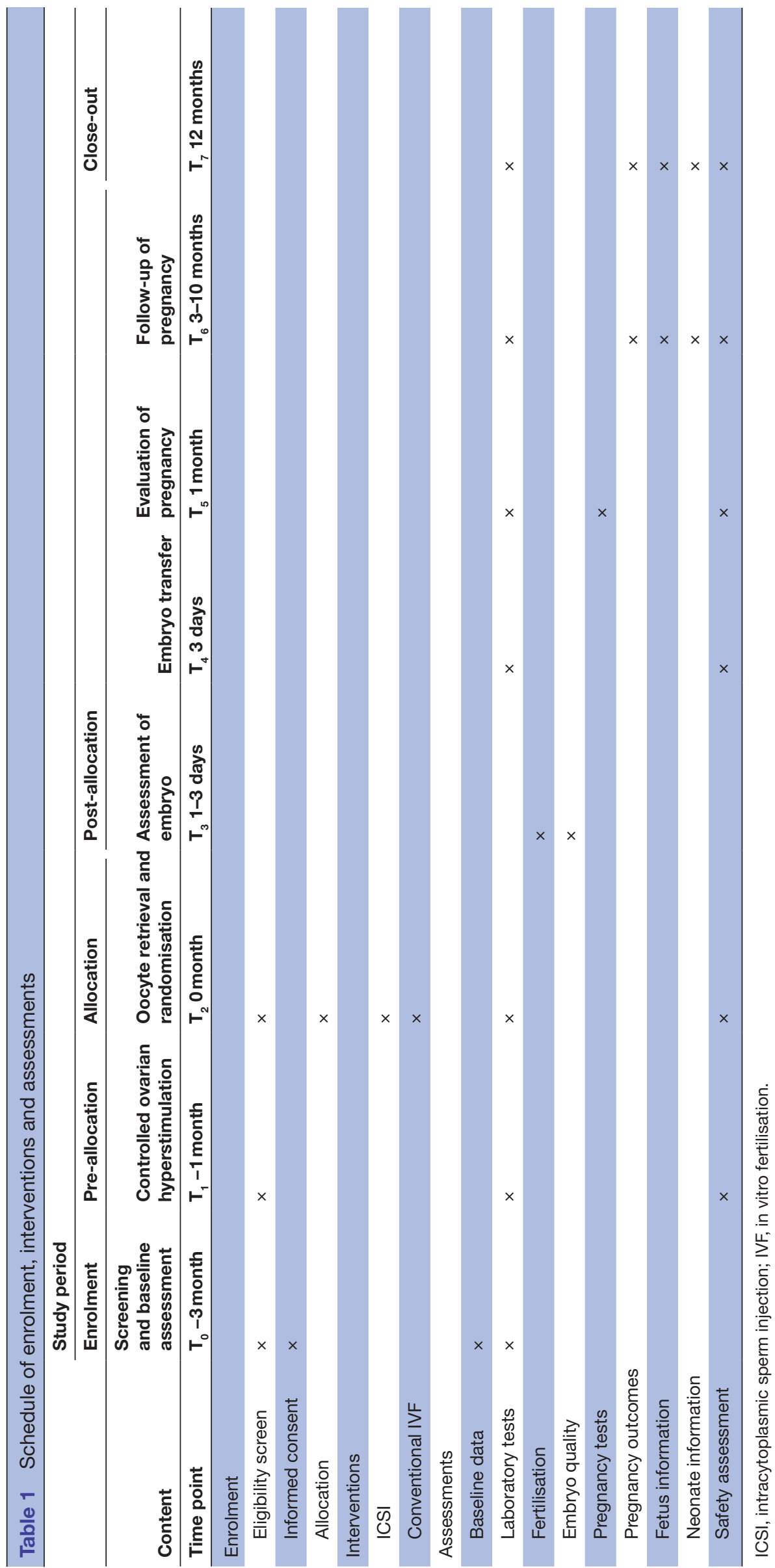

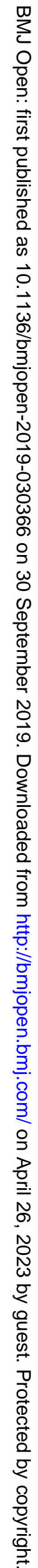


reasons for exclusion will be obtained and stored. On the day of oocyte retrieval, semen of patients who have signed consent form will be analysed again for the exclusion criteria. Ineligible patients will be further excluded from our trial, continuing their conventional clinical procedures instead.

\section{Randomisation}

Randomisation and allocation of eligible patients to study groups will be performed on the day of oocyte retrieval. This procedure will be performed by administrative staffs in the trial centre not involved in the treatment procedure, using an online trial system with a computer-generated randomisation list that allocates couples in a 1:1 ratio to ICSI or IVF, with a variable block size of four or six stratified for centre. Stratified permuted block randomisation will be centrally controlled.

\section{Blinding}

The trial was originally designed and performed as a double-blind trial, in which participants and clinicians/ nurses who performed embryo transfer or follow-up, as well as the investigators and assessors will be blinded until the primary outcome occurred. While embryologists who performed IVF and ICSI were not blinded. Recruitment was slow due to the double-blind design, as participants wanted to know about their allocation of fertilisation method as soon as possible. Therefore, after recruitment of 115 participants, the design was changed to an openlabel study: on the day of oocyte retrieval, administrative staff in the IVF laboratory will log into the trial system to randomise and allocate participants to receive either ICSI or IVF. Initially, only embryologists will know the allocation. Participants and clinicians will be informed about the randomised allocation on the day of embryo transfer for participants with fresh embryo transfers and the day of embryo freezing for couples with freeze-all strategies. Prior to these dates, participants and clinicians will still be unaware of randomisation allocation.

\section{Interventions}

Controlled ovarian hyperstimulation

All couples will receive $\mathrm{COH}$ treatment, which is performed by standard routine according to each study centre. The $\mathrm{COH}$ treatment includes either GnRH-a protocol or GnRH-ant protocol, and the selection of protocol will be done by physicians. In the GnRH-ant protocol, participants will be injected Gonadotropin (Gonal-F or Pouliquen or HMG) daily from cycle day 2 or 3 . When at least one follicle has reached a diameter of $12 \mathrm{~mm}$ or on day 6 of ovarian stimulation, GnRH-ant (Cetrotide or Ganirelix) will be administered subcutaneously until the trigger day (include the trigger day). For super long GnRH-a protocol, GnRH-a (Alarelin or Triptorelin) will be used in previous menstrual cycle, gonadotropin treatment starts after 28-35 days on GnRH agonist downregulation. For long GnRH-a protocol, pituitary downregulation will be initiated 7-10 days before the menstrual cycle with GnRH-a (Alarelin or Triptorelin). After 10-14 days or on day 2 of menstrual cycle, gonadotropin treatment will start. For short GnRH-a protocol, participants will receive Alarelin or Triptorelin for the pituitary downregulation on day 2or3 of menstrual cycle. Gonadotropin will be used on the same time. For above treatments, menstrual cycle of patient includes spontaneous menstrual cycle, and irregular menstrual cycle by the use of oral contraceptives or progestins. Before gonadotrophin treatment, baseline pelvic ultrasound, as well as basic serum hormones (such as FSH, luteinising hormone, progesterone $(\mathrm{P})$ and $\beta$-human chorionic gonadotropin ( $\beta$-hCG) $)$ will be measured to confirm the follicle status. The initial dosage gonadotrophin (Gonal-F or Pouliquen or HMG) is $150-300 \mathrm{mg} /$ day and the subsequent dose will be adjusted according to the individual response. Gonadotrophin treatment will be continued to the trigger day. After two or more follicles reach a diameter $\geq 18 \mathrm{~mm}$, $250 \mu \mathrm{g}$ of hCG (Ovitrelle, $250 \mu \mathrm{g} \mathrm{sc}$ ) will be once injected on trigger day.

\section{Oocyte retrieval and preparation}

Oocyte retrieval is scheduled for 36 hours $( \pm 2)$ after hCG injection. Routine oocyte pick-up is performed under transvaginal ultrasound guidance via 17-18G oocyte aspiration needle with use of intravenous sedation. The retrieved cumulus oocyte complexes (COCs) will be placed in culture medium covered by lightweight paraffin oil and incubated in a humidified $37^{\circ} \mathrm{C}, 5 \% / 6 \% \mathrm{CO}_{2}$ incubator after oocyte retrieval immediately. Besides, the COCs are incubated for 2-6hours before insemination or injection.

\section{Semen preparation}

Fresh ejaculate semen samples will be obtained by masturbation after 2-7 days' abstention from sexual intercourse on the day of oocyte retrieval. Sperm concentration and progressive motility are assessed by computer-assisted semen analysis according to the fifth edition of WHO laboratory standards of human semen and sperm. ${ }^{37}$ All semen samples are prepared by discontinue density gradient centrifugation or swim-up protocol according to local routines. Microscopes (200-400 times) will be used to observe whether there are serious abnormalities in sperm morphology that could lead to fertilisation failure, such as globozoospermia.

\section{ICSI group}

Oocytes in couples allocated to ICSI, will undergo ICSI which has been previously described. ${ }^{38}$ In short, as the enzymatic removal was done in oocyte preparation, the denuded oocytes are examined to assess integrity and maturity. Only those oocytes that have extruded the first polar body (metaphase-II oocytes) will be microinjected.

\section{IVF group}

All the oocytes in couples allocated to IVF will be treated by conventional IVF which is adhered to every study, in which every oocyte will be inseminated by sperm with 
progressive motility concentrate $0.1-0.2 \times 10^{6} \sim 39-42$ hours after hCG injection.

\section{Assessment of fertilisation and embryo quality}

Apart from the fertilisation procedure, assisted reproductive treatments will be similar for the two groups. Assessment of fertilisation is carried out about 16-18 hours (day 1) after fertilisation. Normal fertilisation was assessed by the presence of two pronuclei and a second polar body. The zygotes were cultured in cleavage medium to day 3 , and the cleavage embryo quality will be observed at $67-69$ (day 3) hours after fertilisation. The embryos are scored according to the quality, numbers, size of the blastomeres and the amount of anucleate fragmentation.

\section{Embryo transfer and luteal support}

Fresh or frozen-thawed embryo transfer will be decided by physicians according to conditions of patients. Transfer of fresh embryos is the usual practice when fresh embryos are available in all our study centres. In some cases, all embryos may be cryopreserved without a fresh-embryo transfer, most commonly to prevent the ovarian hyperstimulation syndrome (OHSS). In addition, a freeze-all strategy will be used in the following scenarios: hydrosalpinx, elevated progesterone in hGC day, endometrial factors (endometrial polyps, endometrial cavity fluid and thin endometrium), systematic diseases (stomach-ache, fever or cold) and sudden accident of patients.

Fresh or frozen-thawed embryo transfer will be performed by physicians in 3 or 5 days following the day of oocytes collection for participants receive fresh embryo transfer, and 4-6 days after progesterone initiation for participants undergo frozen-thawed embryo transfer (within 6 months after oocyte aspiration). To reduce the risk of high-order multiple pregnancies, the number of embryos replaced will be limited up to two best-quality embryos in all study centres (one embryo would be transferred if there is uterine malformation, history of uterine surgery or caesarean section). Luteal support, as well as embryo freezing and thawing is performed by standard routines at each study centre, as we assume that the different protocols will be equally distributed in the interventional and control groups.

\section{Follow-up}

Urine and blood hCG will be measured 14 days after embryo transfer, and positive results indicate biochemical pregnancy. If the gestational sac is observed with ultrasonography on 7 weeks of gestation, clinical pregnancy will be confirmed. Ongoing pregnancy is defined by the presence of a gestational sac with fetal heartbeat after 12 weeks of gestation. In 6 weeks after delivery, the information of pregnancy (pregnancy complications and fetus information), delivery information (gestational age, delivery mode, placenta abnormality and/or delivery complications), infant information (such as sex, birth weight, birth defect) will be collected by completing forms designed for this visit.

\section{Outcome measures}

Primary outcome

Our primary outcome will be ongoing pregnancy leading to live birth after the first embryo transfer. Live birth will be defined as a delivery of one or more living infants. ${ }^{17}$

\section{Secondary outcomes}

For the effectiveness of the treatment, we will record these secondary outcomes in terms of effectiveness:

1. Fertilisation: defined as number of zygotes with 2PN (per woman randomised and per oocyte retrieved).

2. Total fertilisation failure: defined as no oocyte formed $2 \mathrm{PN}$ in this given cycle.

3. Available embryo: defined as number of embryos $\geq 4$ cells and $\leq 30 \%$ fragmentation (except embryos developed from $\geq 3$ PN zygotes) on day 3 observation.

4. Good quality embryo: defined as number of embryos with $\geq 6$ cells and $\leq 10 \%$ fragmentation developed from 2PN zygotes on day 3 observation.

5. Implantation: defined as the number of gestational sacs observed per embryo transferred.

6. Clinical pregnancy: defined as one or more observed gestational sac or definitive clinical signs of pregnancy under ultrasonography at 7 weeks of gestation (including clinically documented ectopic pregnancy).

7. Multiple pregnancy: defined as a pregnancy with two or more gestational sacs or positive heart beats at 7 weeks of gestation.

8. Ongoing pregnancy: defined as the presence of a gestational sac and fetal heartbeat after 12 weeks of gestation.

For the safety of the treatment, we will record the following treatment complications as secondary outcomes:

1. Moderate/severe OHSS: defined as exaggerated systemic response to ovarian stimulation characterised by a wide spectrum of clinical and laboratory manifestations. It is classified as mild, moderate or severe according to the degree of abdominal distention, ovarian enlargement, and respiratory, haemodynamic and metabolic complications.

2. Miscarriage: defined as the spontaneous loss of an intra-uterine pregnancy prior to 22 completed weeks of gestational age.

3. Ectopic pregnancy: defined as the implantation takes place outside the uterine cavity, confirmed by sonography or laparoscopy.

We will also collect the following obstetric and perinatal complications:

1. Gestational diabetes mellitus.

2. Hypertensive disorders of pregnancy (comprising pregnancy-induced hypertension, pre-eclampsia and eclampsia).

3. Antepartum haemorrhage, including placenta previa, placenta accreta and unexplained.

4. Preterm birth: defined as birth of a fetus delivered after 22 and before 37 completed weeks of gestational age in participants confirmed ongoing pregnancy. 
5. Birth weight, including low birth weight (defined as weight $<2500 \mathrm{~g}$ at birth), very low birth weight (defined as $<1500 \mathrm{~g}$ at birth), high birth weight (defined as $>4000 \mathrm{~g}$ at birth) and very high birth weight (defined as $>4500 \mathrm{~g}$ at birth).

6. Large for gestational age (defined as a birth weight greater than the 90th centile of the sex-specific birth weight for a given gestational age reference) and small for gestational age (defined as a birth weight less than the 10th centile for gestational age).

7. Congenital anomaly (defined as structural or functional disorders that occur during intrauterine life and can be identified prenatally, at birth or later in life), including trisomy 13, 18, 21, neural tube defect, congenital heart disease, cleft lip, excessive numbers of fingers or toes, hydrocephalus. Clinical diagnosis of congenital anomaly is defined according to the International Classification of Diseases, revision 10 criteria. $^{39}$

8. Perinatal mortality (defined as fetal or neonatal death occurring during late pregnancy (at 222 completed weeks of gestational age and later), during childbirth or up to seven completed days after birth).

9. Neonatal mortality (defined as death of a live born baby within 28 days of birth).

\section{Data management}

The data collected for the trial will be a mixture of routinely clinical data and information from follow-up, which are verifiable from the medical record. To guarantee the authentic study results, all of our researchers and clinicians are required to master all details about this study. All the characteristics in our study are collected at baseline and follow-up through a standard clinical electronic data collection system. All participant-identifiable data, such as consent forms, screening and identification logs will be stored in the investigator site files, accessible only to delegated members of the study team.

Safety reporting will be in accordance with plan and all adverse events will be recorded and informed DSMB. The DSMB will perform an interim analysis 3 months after the first 600 randomised participants have completed embryo transfer. They will do so using the endpoint ongoing pregnancy, as data on live birth will not be available. Also, the DSMB will oversee the severe adverse events (SAEs) that have occurred.

\section{Sample size}

Among couples with non-severe male infertility, the average live birth rate after IVF during 2014-2015 calculated over all study sites was $40 \%$ per cycle. Based on other studies within fertility care as well as the discussion by gynaecologist and methodologists, we assumed that the minimal clinical important difference to make ICSI preferable over IVF would be 7\%. To demonstrate this difference with two-sided test, $5 \%$ alpha-error, $90 \%$ statistical power and taking consideration a dropout of $10 \%$, we will need to enrol 1173 participants in each group, that is, a total of 2346 participants (the ratio between groups will be $1: 1$ ). For the interim analysis, we will use the Haybittle-Peto boundary. The significance level for the interim analysis will be 0.001 and for the final analysis $0.05 .^{40}$

\section{Statistical analysis}

For continues variables, parameters normally distributed will be expressed as mean with $\mathrm{SD}$ and compared using Student's t-test. If the parameters are non-normally distributed, their medians and IQRs will be reported, the Mann-Whitney $\mathrm{U}$ test will be utilised to test the distribution of these variables as well. For categorical variables, we will present the proportion between each group and distributions will be compared using Pearson's $\chi^{2}$ test and Fisher's exact test when appropriate. Data analysis of this trial will follow intention-to-treat principle, in which all randomised women will be considered in the primary comparison between treatment groups. Per-protocol analysis will be conducted as a secondary analysis in participants who complied with protocol.

For missing values, a range of clinically plausible scenarios will be used to impute missing values in order to test the robustness of the findings. For losses to follow-up and protocol violations, we will attempt sensitive analyses to explore the effect of these factors on the trial findings. All tests will be two-tailed, and differences with $\mathrm{p}$ value $<0.001$ for interim analysis or $p$ value $<0.05$ for final analysis are considered statistically significant. All statistical analyses will be performed with the SAS software package V.9.4. The statistical analysis will be done by an independent statistician, overseen by Clinical Epidemiology Research Center of Peking University Third Hospital. The analysis will be described in detail in a statistical analysis plan.

\section{Patient and public involvement}

This research was done without patient or public involvement. Neither patients nor the public were involved in the development of the research question, study design or implementation of this trial. Patients will not be invited to develop patient relevant outcomes or interpret the results, as well as the writing or editing of final manuscript for readability or accuracy. As interventions in our study are both routine procedures during clinical work, burden of the intervention is assessed by patients themselves.

\section{Ethics and dissemination}

The study is registered on ClinicalTrials.gov. Informed consent will be obtained from each participant before randomisation. The researchers will permit trial-related monitoring, audits, regulatory inspections, providing direct access to source data and documents. There are no additional data available in this study protocol.

\section{Trial status}

The recruitment in each study centre started in April 2018. The estimated end date of the last recruitment for this study is April 2020. 


\section{Author affiliations}

${ }^{1}$ Centre for Reproductive Medicine, Department of Obstetrics and Gynecology, Peking University Third Hospital, Beijing, China

${ }^{2}$ National Clinical Research Centre for Obstetrics and Gynecology, Beijing, China ${ }^{3}$ Key Laboratory of Assisted Reproduction (Peking University), Ministry of Education, Beijing, China

${ }^{4}$ Beijing Key Laboratory of Reproductive Endocrinology and Assisted Reproductive Technology, Beijing, China

${ }^{5}$ Beijing Advanced Innovation Centre for Genomics, Beijing, China

${ }^{6}$ Peking-Tsinghua Centre for Life Sciences, Peking University, Beijing, China ${ }^{7}$ Research Centre of Clinical Epidemiology, Peking University Third Hospital, Beijing, China

${ }^{8}$ Women's Hospital, Zhejiang University School of Medicine, Hangzhou, China

${ }^{9}$ Reproductive Medicine Centre of The Sixth Affiliated Hospital of Sun Yat-Sen University, Guangzhou, China

${ }^{10}$ First Affiliated Hospital of Kunming Medical University, Kunming, China

${ }^{11}$ Haidian Maternal and Child Health Hospital, Beijing, China

${ }^{12}$ First Affiliated Hospital of Anhui Medical University, Hefei, China

${ }^{13}$ The Second Hospital of Hebei Medical University, Shijiazhuang, China

${ }^{14}$ The Third Affiliated Hospital of Guangzhou Medical University, Guangzhou, China

${ }^{15}$ General Hospital of Ningxia Medical University, Yinchuan, China

${ }^{16}$ Robinson Research Institute and Adelaide Medical School, Adelaide, South

Australia, Australia

${ }^{17}$ Department of Obstetrics and Gynaecology, Monash University, Monash Medical Centre, Melbourne, Victoria, Australia

${ }^{18}$ The International Peace Maternity and Child Health Hospital, School of Medicine, Shanghai Jiao Tong University, Shanghai, China

Contributors JQ, H-FH, LR, RW and BWM conceived the study idea. JQ, H-FH, LR, RY, YL and DZ participated in the design of the study, recruitment of participants and drafting of the manuscript. $\mathrm{DZ}$ and RY participate in recruitment of participants and assessment of clinical outcomes. JQ, H-FH, YZ, XL, LT, HW, YC, GH, JL and JZ supervised patient diagnosis and recruitment in each study centre. DZ and LZ coordinates of the data collection. LZ oversees data collection and will performed data analysis. DZ, LZ, RW and BWM will design the statistical analysis plan and oversee statistical analysis. YL oversees laboratory work among 10 centres. All authors critically reviewed the article and approved the final manuscript.

Funding This study was supported by National Key Research and Development Program of China (2016YFC1000201; 2018YFC1002104) and the National Science Foundation of China (81730038). The study funders had no rule in the study design, implementation, analysis, manuscript, preparation or decision to submit this article for publication.

\section{Competing interests None declared.}

Patient consent for publication Not required.

Ethics approval This trial had been reviewed and approved by the medical science research ethics committee of Peking University Third Hospital: D2017050.

Provenance and peer review Not commissioned; externally peer reviewed.

Open access This is an open access article distributed in accordance with the Creative Commons Attribution Non Commercial (CC BY-NC 4.0) license, which permits others to distribute, remix, adapt, build upon this work non-commercially, and license their derivative works on different terms, provided the original work is properly cited, appropriate credit is given, any changes made indicated, and the use is non-commercial. See: http://creativecommons.org/licenses/by-nc/4.0/.

ORCID iD

Lin Zeng http://orcid.org/0000-0001-8707-5854

\section{REFERENCES}

1. Krausz C. Male infertility: pathogenesis and clinical diagnosis. Best Pract Res Clin Endocrinol Metab 2011;25:271-85.

2. Hull MG, Glazener CM, Kelly NJ, et al. Population study of causes, treatment, and outcome of infertility. BMJ 1985;291:1693-7.

3. Crosignani PG, Walters DE. Clinical pregnancy and male subfertility; the ESHRE multicentre trial on the treatment of male subfertility. European Society of human reproduction and embryology. Hum Reprod 1994;9:1112-8.
4. O'Donovan PA, Vandekerckhove P, Lilford RJ, et al. Infertility:Treatment of male infertility: is it effective? Review and meta-analyses of published randomized controlled trials. Hum Reprod 1993;8:1209-22.

5. Tournaye H. Male factor infertility and ART. Asian J Androl 2012;14:103-8.

6. Cohen J, Fehilly C, Fishel S, et al. Male infertility successfully treated by in-vitro fertilisation. The Lancet 1984;323:1239-40.

7. Fishel S, Aslam I, Lisi F, et al. Should ICSI be the treatment of choice for all cases of in-vitro conception? Hum Reprod 2000;15:1278-83.

8. Devroey P, Van Steirteghem A. A review of ten years experience of ICSI. Hum Reprod Update 2004:10:19-28.

9. Palermo Get al. Pregnancies after intracytoplasmic injection of single spermatozoon into an oocyte. The Lancet 1992;340:17-18.

10. Coates TE, Check JH, Choe J, et al. An evaluation of couples with failure of fertilization in vitro. Hum Reprod 1992;7:978-81.

11. Aboulghar MA, Mansour RT, Serour Gl, et al. Management of longstanding unexplained infertility: a prospective study. Am J Obstet Gynecol 1999;181:371-5.

12. Fan W, wei Li S, Li L, et al. Outcome of conventional IVF and ICSI on sibling oocytes in the case of isolated teratozoospermia. $J$ Assist Reprod Genet 2012;29:905-10.

13. Xie B, Zhu W-jie, Huang Y-hua. Outcome of conventional IVF and ICSI on sibling oocytesin moderate oligoasthenozoospermia. Pak J Med Sci 2013;29:1221-4.

14. Palermo G, Joris H, Derde MP, et al. Sperm characteristics and outcome of human assisted fertilization by subzonal insemination and intracytoplasmic sperm injection. Fertil Steril 1993;59:826-35.

15. Verheyen G, Tournaye H, Staessen $\mathrm{C}$, et al. Controlled comparison of conventional in-vitro fertilization and intracytoplasmic sperm injection in patients with asthenozoospermia. Hum Reprod 1999;14:2313-9.

16. Payne D, Flaherty SP, Jeffrey R, et al. Andrology: successful treatment of severe male factor infertility in 100 consecutive cycles using intracytoplasmic sperm injection. Hum Reprod 1994;9:2051-7.

17. Zegers-Hochschild F, Adamson GD, Dyer S, et al. The International glossary on infertility and fertility care, 2017. Fertil Steril 2017; 108:393-406

18. Dyer S, Chambers GM, de Mouzon J, et al. International committee for monitoring assisted reproductive technologies world report: assisted reproductive technology 2008, 2009 and 2010. Hum. Reprod. 2016;31:1588-609.

19. Boulet SL, Mehta A, Kissin DM, et al. Trends in use of and reproductive outcomes associated with intracytoplasmic sperm injection. JAMA 2015;313:255-63.

20. Blake M, Garrisi J, Tomkin G, et al. Sperm deposition site during ICS affects fertilization and development. Fertil Steril 2000;73:31-7.

21. Terada Y, Luetjens CM, Sutovsky P, et al. Atypical decondensation of the sperm nucleus, delayed replication of the male genome, and sex chromosome positioning following intracytoplasmic human sperm injection (ICSI) into golden hamster eggs: does ICSI itself introduce chromosomal anomalies? Fertil Steril 2000;74:454-60.

22. Miller JE, Smith TT. The effect of intracytoplasmic sperm injection and semen parameters on blastocyst development in vitro. Hum Reprod 2001;16:918-24.

23. Lie RT, Lyngstadass A, Ørstavik KH, et al. Birth defects in children conceived by ICSI compared with children conceived by other IVFmethods; a meta-analysis. Int J Epidemiol 2005;34:696-701.

24. Wen J, Jiang J, Ding C, et al. Birth defects in children conceived by in vitro fertilization and intracytoplasmic sperm injection: a metaanalysis. Fertil Steril 2012;97:1331-7.

25. Eftekhar M, Mohammadian F, Yousefnejad F, et al. Comparison of conventional IVF versus ICSI in non-male factor, normoresponder patients. Iran J Reprod Med 2012;10:131-6.

26. Kim JY, Kim JH, Jee BC, et al. Can intracytoplasmic sperm injection prevent total fertilization failure and enhance embryo quality in patients with non-male factor infertility? Eur J Obstet Gynecol Reprod Biol 2014;178:188-91.

27. Park JH, Jee BC, Kim SH. Comparison of normal and abnormal fertilization of in vitro -matured human oocyte according to insemination method. J. Obstet. Gynaecol. Res. 2016;42:417-21.

28. Tannus $\mathrm{S}$, Son $\mathrm{W}-\mathrm{Y}$, Gilman A, et al. The role of intracytoplasmic sperm injection in non-male factor infertility in advanced maternal age. Hum Reprod 2017;32:119-24

29. Pisarska MD, Casson PR, Cisneros PL, et al. Fertilization after standard in vitro fertilization versus intracytoplasmic sperm injection in subfertile males using sibling oocytes. Fertil Steril 1999;71:627-32.

30. Elizur SE, Levron J, Seidman DS, et al. Conventional in vitro fertilization versus intracytoplasmic sperm injection for sibling oocytes in couples with mild oligoteratoasthenozoospermia and couples with normal sperm. Fertil Steril 2004;82:241-3. 
31. van der Westerlaken L, Naaktgeboren $\mathrm{N}$, Verburg $\mathrm{H}$, et al. Conventional in vitro fertilization versus intracytoplasmic sperm injection in patients with borderline semen: a randomized study using sibling oocytes. Fertil Steril 2006;85:395-400.

32. Hamberger L, Sjögren A, Lundin K, et al. Microfertilization techniques--the Swedish experience. Reprod Fertil Dev 1995;7:263-7.

33. Bhattacharya S, Hamilton MPR, Shaaban M, et al. Conventional in-vitro fertilisation versus intracytoplasmic sperm injection for the treatment of non-male-factor infertility: a randomised controlled trial. The Lancet 2001;357:2075-9.

34. Hershlag A, Paine T, Kvapil G, et al. In vitro fertilizationintracytoplasmic sperm injection split: an insemination method to prevent fertilization failure. Fertil Steril 2002;77:229-32.

35. Qiao J, Feng HL. Assisted reproductive technology in China: compliance and non-compliance. Transl Pediatr 2014;3:91-7.
36. Chan A-W, Tetzlaff JM, Altman DG, et al. Spirit 2013 statement: defining standard protocol items for clinical trials. Ann Intern Med 2013;158:200.

37. Anon. Who manual for the examination and processing of human semen. Geneva: WHO, 2010.

38. Oehninger S, Veeck L, Lanzendorf S, et al. Intracytoplasmic sperm injection: achievement of high pregnancy rates in couples with severe male factor infertility is dependent primarily upon female and not male factors. Fertil Steril 1995;64:977-81.

39. Anon. International statistical classification of diseases and related health problems. 10th revision. Geneva: WHO, 2016.

40. Blenkinsop A, Parmar MK, Choodari-Oskooei B. Assessing the impact of efficacy stopping rules on the error rates under the multiarm multi-stage framework. Clin Trials 2019;1496049025. 